\title{
13. Fazit: Chruschtschows Vorstellungen und das daraus erwachsende Vorgehen
}

\author{
Konzept des Vorgehens in der Krise
}

Chruschtschow traf, ohne vorher Rat einzuholen und Bedenken im eigenen Lager zu beachten, persönlich die Entscheidung, die Westmächte im November 1958 mit dem Berlin-Ultimatum zu konfrontieren, und löste damit eine der gefährlichsten Krisen im Kalten Krieg aus. Zwar spitzte sich die Auseinandersetzung nicht so dramatisch zu wie während der Kuba-Krise, aber dafür wurden die Widersacher ungewöhnlich lange in Atem gehalten. Die Folgen bestimmten die Situation in Deutschland bis zum Ende des Ost-West-Konflikts. Chruschtschow blieb während der gesamten Dauer der Krise die treibende Kraft und war stets der entscheidende Initiator des Geschehens, während die Leiter der westlichen Politik weithin nur reagierten. Das bleibende Resultat war die Berliner Mauer. Der sowjetische Führer hatte freilich noch mehr im Blick gehabt: die Erzwingung eines Friedensvertrages, der die Teilung Deutschlands vollenden sollte, und die Aufhebung der westlichen Besatzungsrechte, die West-Berlin in ein abhängiges Gebiet verwandeln sollte. Daran hielt er nach dem Mauerbau weiter fest. Das wird in manchen Darstellungen übersehen, welche die Sperrung der Sektorengrenze als Abschluß wählen und diese als Endpunkt betrachten. Tatsächlich erreichte die Krise im folgenden Herbst den Höhepunkt und flaute erst danach allmählich unter wiederholtem Aufflackern ab.

Die Hartnäckigkeit, mit der Chruschtschow bis zum Schluß auf der uneingeschränkten Erfüllung der Forderungen bestand, ließ ihn trotz weitreichender Konzessionsbereitschaft auf westlicher Seite, vor allem bei Kennedy, nichts erreichen außer der einseitig durchgeführten, von den Westmächten ohne wesentlichen Widerstand hingenommenen Abriegelung West-Berlins, die er lange von sich gewiesen hatte und zunächst nur als zeitweilige Notmaßnahme betrachtete. Der Ablehnung aller Kompromisse lag die ideologisch bedingte Einschätzung zugrunde, das Kräfteverhältnis werde sich unaufhaltsam zugunsten des sozialistischen Lagers verändern. Wenn er nur die eigene Position ungemindert aufrechterhalte, werde der UdSSR früher oder später von selbst zufallen, was sie verlange, statt sich mit teilweiser Erfüllung ihrer Forderungen begnügen zu müssen. Wie er meinte, blieb der Erfolg im Ringen um Berlin nicht aus, sondern verzögerte sich nur.

Auch sonst ließ sich Chruschtschow vielfach von Vorstellungen leiten, die sich in der Realität nicht bewährten. Als er 1958 die direkte Attacke gegen West-Berlin eröffnete, glaubte er die westlichen Regierungen mit dem Argument beeindrucken zu können, die dortige Position biete ihnen keinen Vorteil, sondern nütze nur den gefährlichen Revanchepolitikern in Bonn. Wieso sollten sie deswegen Risiken ein- 
gehen? Er wußte freilich sehr wohl, daß es nicht um die westliche Exklave als Machtfaktor ging. Die Stadt war vielmehr das symbolische Unterpfand des amerikanischen Engagements für Westeuropa und damit ein entscheidendes Element des NATO-Zusammenhalts. Die Absicht, diese Grundlage der westlichen Sicherheit zu erschüttern, war ein wichtiges Motiv seines Vorgehens. Es ist unerfindlich, wieso er hoffte, führende westliche Politiker über diesen Zusammenhang täuschen zu können, der selbst ihm als Außenstehendem klar war.

Chruschtschow Sicht der Lage in Deutschland ließ ebenfalls Realitätssinn vermissen. Völlig abwegig war die Erwartung, daß er die Deutschen, vor allem die West-Berliner, durch den Vorschlag einer „Freien Stadt“ zumindest längerfristig davon überzeugen könnte, daß der Verzicht auf die Bindung an den Westen und die Annahme seiner „Angebote“ vorteilhaft sei. Die Aussicht, der Sowjetmacht ohne die Sicherheitsgewähr von USA und NATO gegenüberzustehen und sich statt dessen auf Versprechungen der UdSSR und der DDR verlassen zu müssen, erschien vor allem angesichts des Systemantagonismus bedrohlich. Für die WestBerliner war die Aussicht, auf die Verbindung zur westlichen Wohlstandszone und die Subventionen aus Bonn zu verzichten, um sich in Abhängigkeit vom Warenaustausch mit den - angeblich im Gegensatz zum Westen krisenfreien - sozialistischen Staaten zu begeben, alles andere als attraktiv.

Chruschtschow wähnte, der Sozialismus sei dem Kapitalismus überlegen und könne den Wettstreit der Systeme in Deutschland gewinnen. Das Gegenteil war der Fall. Die DDR erwies sich ständig als ungleich weniger leistungsfähig als die Bundesrepublik. Ulbricht führte das auf eine angeblich nachteilige Ausgangssituation und die offene Westgrenze zurück. Zunächst versagte Chruschtschow der Grenzschließung seine Genehmigung; die Voraussetzungen für den Erfolg im intersystemaren Wettbewerb sollten mit sowjetischer Hilfe geschaffen werden. Als das nicht zum Erfolg führte, rang er sich dazu durch, das Problem durch Ausschaltung der vermeintlich daran hindernden Einflüsse aus dem Westen zu lösen. Aber auch das ließ die DDR nicht ökonomisch gedeihen. Ihre Wirtschaft blieb nach wie vor erheblich zurück und vermochte trotz weiterer Unterstützung der UdSSR ihre Abhängigkeit von westdeutschen Lieferungen nicht zu überwinden. Das damit fortdauernde Defizit an ökonomischer Standfestigkeit trug sehr wesentlich dazu bei, daß Chruschtschow darauf verzichtete, den Westen durch Abschluß eines separaten Friedensvertrages mit der DDR und die damit verknüpfte ostdeutsche Kontrolle über die Zugangswege herauszufordern. Aller Rückschläge ungeachtet, hielt er aber an der grundsätzlichen Gewißheit fest, daß der Sozialismus das effizientere System sei. Er meinte daher, der Sieg des Sozialismus im Wettbewerb mit dem Kapitalismus auf deutschem Boden werde sich lediglich verzögern.

\section{Haltung gegenüber den Westmächten}

Chruschtschow war zuversichtlich, den Westmächten durch die angedrohte Übergabe der West-Berliner Zugangswege an die DDR das Kriegsrisiko zuschieben zu können. Das würden sie ebenso wenig wie die UdSSR eingehen wollen 
und sich daher zur Erfüllung seiner Forderungen bereit finden. Das war eine sehr kühne Erwartung. Hätte die Eisenhower-Administration nicht Rücksicht auf die westeuropäischen Verbündeten genommen, die im Kriegsfall anders als die USA eine weitgehende nukleare Vernichtung befürchten mußten, wäre das Spiel mit den Ängsten im Westen möglicherweise bereits vor dem formellen Ultimatum vom 27. November $1958 \mathrm{zu}$ Ende gewesen. Aber auch nachdem sich die westlichen Regierungen zu Verhandlungen über die sowjetischen Forderungen veranlaßt gesehen hatten, um die angedrohte Maßnahme abzuwenden, ging die Rechnung nicht auf. Die Amerikaner wollten sich nicht zu Schritten der Beschwichtigung drängen lassen. Westliche Politiker wie vor allem der britische Premierminister Macmillan, die der UdSSR sehr weit entgegenzukommen bereit waren, sahen sich durch den Zwang zum innerwestlichen Konsens gebremst. Differenzen im Bündnis verhinderten in einigen Fällen sogar, daß überhaupt verbindliche Stellungnahmen abgegeben werden konnten, so daß Chruschtschow auch dadurch die angestrebte Übereinkunft verfehlte.

Anders als Macmillan vertrat der französische Staatspräsident de Gaulle den Standpunkt, man solle nicht mit einer Macht verhandeln, die Druck ausübe und nur ein im voraus festgelegtes Ergebnis akzeptieren wolle. Auch wenn er im Gegensatz zu den Amerikanern keine Abwehrmaßnahmen traf, hielt er es für richtig, auf die sowjetischen Forderungen nach Änderung des Berlin-Status nicht einzugehen. Obwohl Chruschtschow diese Haltung bei Adenauer schärfstens verurteilte, war er gegenüber de Gaulle nachsichtig. Frankreich war psychologisch-politisch in einer ungleich besseren Lage als die durch die Vergangenheit belastete Bundesrepublik. Moralische Diffamierung und propagandistischer Druck versprachen daher in seinem Falle keinen Erfolg. Zudem glaubte der Kremlchef, einen gemeinsamen Nenner mit dem französischen Staatspräsidenten gefunden zu haben: Er hielt ihn für einen Gegner der deutschen Einheit und sah erste Anzeichen für einen Haltungswandel gegenüber der NATO. Auch die Einsicht, daß man die Positionen des Sozialismus akzeptieren müsse, unterscheide ihn von den „reaktionären“ Politikern in Bonn und Washington.

Die sowjetische Seite erklärte immer wieder, daß ihre Forderungen lediglich darauf hinausliefen, den Status quo vor Angriffen aggressiver Kräfte zu schützen. Der Wille westlicher Führer wie Macmillan und später auch Kennedy zu Vereinbarungen auf der Grundlage des Bestehenden sollte sie demnach dazu veranlassen, den sowjetischen Forderungen zustimmen. Dabei wurde übersehen, daß diese Politiker zwar für den politischen Status quo eintraten, aber keineswegs bereit waren, diesen zur Basis rechtlicher Veränderungen zu machen, die politische Veränderungen nach sich zogen. Aufgrund der fehlenden Differenzierung äußerte Chruschtschow intern, Kennedy halte den Konflikt um West-Berlin nur darum aufrecht, um Spannung zu erzeugen, welche die Westdeutschen an die NATO bänden. Daß er den amerikanischen Willen zur Behauptung der Stadt nicht ernstnahm, zeigt auch seine Ansicht, dem Streit lägen wesentlich Prestigebedürfnisse der anderen Seite zugrunde. Berücksichtige man diese, etwa indem man eine gesichtswahrende Interimsregelung vorschlage, war demnach eine Einigung zu erzielen, ohne daß sich die UdSSR auf einen echten Kompromiß einzulassen brauchte. Weil man meinte, alles bekommen zu können, wies man selbst weitrei- 
chende Angebote zurück, obwohl diese als Ansatzpunkte für weitere Zugeständnisse hätten dienen können. Hätte man sofort zugegriffen und ein grundsätzliches Einvernehmen hergestellt, wäre es für Widersacher im Westen schwer gewesen, das Ergebnis wieder in Frage zu stellen. Durch seine Unnachgiebigkeit förderte der Kreml die totale Ablehnung seiner Forderungen.

Chruschtschow hielt jedes ernstliche Eingehen auf westliche Vorstellungen und Interessen auch darum für unangebracht, weil er aufgrund der marxistisch-leninistischen Ideologie davon überzeugt war, daß sich das internationale Kräfteverhältnis fortlaufend zugunsten des Sozialismus verschiebe. Er glaubte, abwarten zu können, bis sich die andere Seite zur Annahme der gestellten Forderungen gezwungen sehe. Die sowjetischen Unterhändler verlangten daher vom Westen, er müsse zuerst ihre Vorschläge zu Friedensvertrag und Freistadt vollständig und verbindlich akzeptieren, ehe Gespräche über eine Regelung des ihn vor allem anderen interessierenden Zugangsproblems geführt werden könnten. Zugleich gaben sie zu erkennen, daß jede Vereinbarung darüber, auf einer uneingeschränkten, nicht nur formalen Anerkennung der DDR-Souveränität zu beruhen habe. $\mathrm{Zu}$ einer solchen Kapitulation mochte sich kein westlicher Führer verstehen.

\section{Entwicklungen im Verhältnis zu den Westmächten}

Nach Scheitern des Versuchs zur Durchsetzung der sowjetischen Forderungen während der Präsidentschaft Eisenhowers, mit dem überdies zuletzt das persönliche Verhältnis durch die U-2-Affäre zerrüttet war, setzte Chruschtschow seine Hoffnung auf dessen Nachfolger Kennedy. In seiner Sicht, die er durch den Verlauf der Wiener Begegnung bestätigt sah, war das ein schwacher Mann, mit dem fertigzuwerden geringere Mühe bereitete. Daher machte er einen zweiten Anlauf mit einem neuen Ultimatum, das den gleichen Inhalt hatte wie das erste. Als Kennedy erkannte, daß Chruschtschow seinen Behauptungswillen bezweifelte, machte er große Anstrengungen, um ihn sowohl durch eine glasklare Darlegung seiner politischen Absichten als auch durch Rüstungs- und Mobilisierungsmaßnahmen von seiner Entschlossenheit zur Verteidigung West-Berlins zu überzeugen. Das erschütterte die Gewißheit des Kremlchefs, seine Forderungen ohne Kriegsrisiko durchsetzen zu können. Das änderte freilich nicht die Einschätzung von Kennedys Persönlichkeit, sondern weckte die Sorge, die „reaktionären“, „militaristischen" Kräfte in den USA könnten Macht über den Präsidenten gewonnen haben. Das wäre für die UdSSR eine außerordentlich gefährliche Situation. Chruschtschow entschloß sich, zunächst die Sektorengrenze zu schließen. Das werde die Amerikaner, die sich auf die Verteidigung allein West-Berlins festgelegt hatten, nicht herausfordern.

Zwar erfüllte sich diese Erwartung, doch ging das Kalkül nicht auf, man werde damit eine gute Ausgangsposition für die Durchsetzung des Friedensvertrags und der Freistadtregelung gewinnen. Der Versuch mißlang, die USA mit vorgeblich überlegener Militärmacht zu beeindrucken und daraus die Gewißheit zu schöpfen, daß Kennedy es unter keinen Umständen auf einen bewaffneten Konflikt ankommen lassen werde. Chruschtschow mußte mit dem Risiko eines Nuklearkrie- 
ges rechnen. Da er dieses keinesfalls eingehen wollte, rückte er vom Termin für den Abschluß des Friedensvertrags am Jahresende ab, hoffte aber zunächst noch, das gesteckte Ziel durch ein weniger hartes, auf Allmählichkeit abgestelltes Vorgehen zu erreichen. Kennedy glaubte freilich, der Kreml sei zur Aushandlung eines Kompromisses bereit, und faßte im Verlauf der daraufhin eingeleiteten Gespräche Zugeständnisse ins Auge, die in ihrer faktischen Auswirkung West-Berlin gefährdet hätten. Er verstand sich freilich entgegen sowjetischem Wunsch nur zu Vorgesprächen, um sich nicht dem Vorwurf der Verbündeten auszusetzen, er wolle ihnen ein Verhandlungsergebnis aufzwingen. Dadurch wurden endgültige Festlegungen gegenüber dem Kreml von vornherein vermieden. Das erlaubte es den anderen betroffenen westlichen Staaten, auf Korrekturen der amerikanischen Haltung zu dringen. Adenauer griff im Frühjahr 1962 in die laufenden Gespräche ein, um eine zur Erörterung stehende Regelung zu Fall zu bringen. Er hatte den französischen Staatspräsidenten de Gaulle auf seiner Seite, so daß er Kennedy nicht isoliert gegenüberstand. Dieser konnte den Einspruch nicht ignorieren, ohne den Zusammenhalt der NATO auf's Spiel zu setzen.

Der Einfluß, den die westeuropäischen Staaten auf die amerikanische Führungsmacht ausübten, war freilich dem an Alleinentscheidung im sozialistischen Lager gewöhnten Kremlchef unverständlich. Chruschtschow suchte zwar die Nachgiebigkeit eines Macmillan gegen die Amerikaner zu instrumentalisieren, wenn diese die Erfüllung seiner Forderungen verweigerten, nahm es aber kaum ernst, wenn Bonn und Paris in Washington auf einen härteren Kurs drangen. Wer wie Adenauer die Vorschläge des Kreml kritisierte und ablehnte, war zu ignorieren. Nur wer Sympathie für sie zu hegen schien wie der westdeutsche Botschafter Kroll, war der Aufmerksamkeit wert. Der Grund für die Fehleinschätzung der politischen Gewichte im westlichen Bündnis war nicht unzureichende Information. Durch die Auslandsaufklärung war Chruschtschow über die Akteure im Westen und ihre unterschiedlichen Standpunkte ausgezeichnet unterrichtet. Was fehlte, war die Fähigkeit, die Fakten angemessen zu interpretieren. Wie wenig er die Zusammenhänge verstand, zeigte sich erneut, als er angesichts der Wirkung, die Adenauer im Frühjahr 1962 mit seinem Einspruch gegen Kennedys Nachgiebigkeit in der Frage des West-Berlin-Zugangs erzielte, die Bedeutung der westdeutschen Haltung nicht länger übersehen konnte: Er reagierte mit dem Versuch, die USA durch überbordende Polemik gegen die „revanchistische Adenauer-Regierung “ umzustimmen, ohne zu sehen, daß er in Washington die Solidarität mit einem wichtigen Bundesgenossen herausforderte, der dort zudem hohe persönliche Wertschätzung genoß.

Durch die amerikanische Entschlossenheit während der Kuba-Krise wurde Chruschtschow davon überzeugt, daß er keine Konfrontation riskieren konnte. Er kam auch je länger, desto mehr zu der Auffassung, daß die alleinige Konzentration der Westpolitik auf Berlin, die ungeachtet des in Verhandlungen und vor Ort ausgeübten Drucks erfolglos geblieben war, an der Verfolgung anderer wichtiger Interessen hinderte. Vor allem schienen Ergebnisse im Abrüstungsdialog mit Washington notwendig. Es galt daher, die Attacken gegen die Westmächte zu beenden, ohne jedoch die Ziele in Berlin aufzugeben. Um beiden Erfordernissen zu entsprechen, wurde ein Konzept entwickelt, das die Forderungen aktuell gegen 
die Bundesrepublik richtete und die langfristig auf Beseitigung der westlichen Präsenz abzielenden Implikationen verschleierte. Die Vier-Mächte-Rechte wurden als Grundlage des Besatzungsregimes in West-Berlin anerkannt, aber zugleich so formuliert, daß sich daraus Folgerungen ergaben, welche die Position der Westmächte allmählich unterminieren sollten. Anders als von Chruschtschow beabsichtigt, ermöglichte die Bereitschaft, die Vier-Mächte-Rechte überhaupt zu akzeptieren, später die Aushandlung der Modus-vivendi-Regelung von 1971. Diese beruhte auf der Respektierung der westlichen Präsenz, des essentiellen Kerns der Bindungen und der sowjetischen Gewähr dafür, daß bei der Abfertigung des zivilen Zugangsverkehrs durch die DDR die Erfordernisse der Lebensfähigkeit WestBerlins gewahrt blieben. Diese Funktion hätte der Kreml nicht übernehmen können, wenn er nicht vom Standpunkt der unbeschränkten DDR-Souveränität abgerückt wäre.

\section{Spannungen im Verhältnis zur DDR}

Die DDR war für Chruschtschow der im Zusammenhang mit der Berlin-Frage weitaus wichtigste Verbündete. Sie war der Schauplatz des Handelns. Einschlägige Funktionen wurden von ihr schon ausgeübt (wie vor allem die Abfertigung des zivilen Transitverkehrs); die damit verbundenen sowie weitere berlinpolitische Entscheidungsbefugnisse sollten ihr durch den Friedensvertrag übertragen werden (wie namentlich die unbeschränkte Souveränität in allen Zugangsfragen und die Befähigung zu Eingriffen in innere Angelegenheiten der „Freien Stadt West-Berlin“). Die ostdeutsche Seite wurde daher in die sowjetischen Aktionspläne einbezogen. Dementsprechend mußte die SED-Führung darüber eingehend unterrichtet werden. Es kam vielfach zu ausführlichen Unterredungen und Briefwechseln, bei denen Ulbricht seine Auffassungen äußern und vertreten konnte. Dabei ging es mindestens ebenso sehr um seine Sache wie um die Chruschtschows. Unerwartet für beide Seiten, die von der Übereinstimmung ihrer Interessen ausgingen, entwickelten sich parallel zum Hauptkonflikt mit den Westmächten Nebenkonflikte zwischen UdSSR und DDR.

Als Chruschtschow im November 1958 Forderungen aufstellte, deren Erfüllung die ostdeutsche Seite von allen Souveränitätsbeschränkungen befreien würde, war er sich offensichtlich zunächst nicht bewußt, daß sich der damit zugestandene Anspruch auf unabhängige Entscheidung auf das Verhältnis zur Sowjetunion auswirken könnte. Allem Anschein nach meinte er, daß die politische Gemeinsamkeit gegenüber dem Westen, die identischen ideologischen Prinzipien und die Solidaritätsverpflichtungen aufgrund des „sozialistischen Internationalismus“ die Einmütigkeit gewährleisteten. Zudem erwartete er, daß der Abschluß des Friedensvertrags rasch zustande kommen werde. Daher würden auch die Folgeregelungen bald wirksam werden. Als das nicht der Fall war, entstand das unvorhergesehene Problem, daß die SED-Führung mit den ihr zugesicherten Souveränitätskompetenzen ernstmachen wollte, der Kreml ihr aber das nicht zugestand, wenn das die Negierung westlicher Rechte bedeutete. In Moskau hielt man es für erforderlich, damit zu warten, bis als notwendige Grundlage der Friedensvertrag 
geschlossen sei. Je länger sich dessen Durchsetzung verzögerte, desto ungeduldiger wurde Ulbricht.

Dazu kamen grundsätzliche Differenzen. Ulbricht setzte gegenüber dem Westen generell nicht auf werbende Lockung, sondern auf die Anwendung von Zwang. Zudem war er im Unterschied zu Chruschtschow, der stets eine breite Palette sowjetischer Interessen im Auge behalten mußte, auf seine Berlin- und Deutschland-Ambitionen fixiert. Erschwerend kam hinzu, daß diese in aller Regel nicht durch eigene Anstrengungen, sondern nur durch die Macht der UdSSR durchzusetzen waren. Daher war Ulbricht auf den Kreml angewiesen, der den Einsatz zu leisten hatte und daher im Unterschied zu ihm die Vor- und Nachteile, Chancen und Risiken des Handelns abwägen mußte. Aus diesen Gründen drängte der ostdeutsche Parteichef, den Worten müßten endlich Taten folgen, während man in Moskau Vorsicht im Umgang mit den Westmächten für notwendig hielt. Man wollte keine Reaktionen hervorrufen, die entweder eigene Interessen tangierten oder unerwünschte Konflikte herbeiführten. So verweigerte man ein Vorgehen gegen die westlichen Militärmissionen in der DDR, weil die Tätigkeit der sowjetischen Militärmissionen in der Bundesrepublik nicht gefährdet werden sollte. Maßnahmen, in deren Folge Auseinandersetzungen mit den Westmächten erwartet wurden, wurden ebenfalls abgelehnt. Nur was keine ernstlichen Risiken in sich barg, war annehmbar.

Ulbricht erhob daraufhin den Vorwurf, der zugesicherte Anspruch auf uneingeschränkte DDR-Souveränität werde mißachtet. Auf die sowjetische Weigerung hin, die Forderung nach Schritten zum Abschluß des vorgesehenen separaten Friedensvertrags zu erfüllen, suchte er den Kreml immer wieder zu Einzelakten zu bewegen, die als Beweis für die Verwirklichung des Souveränitätsanspruchs herhalten konnten. Wenn der Kreml dazu nicht bereit war, bemühte er sich statt dessen um die Genehmigung von DDR-Maßnahmen. Dabei ging es fast ausnahmslos um kleine Dinge, sollte doch die sowjetische Seite ungeachtet ihres Widerstandes gegen die Durchsetzung einer generellen Regelung wenigstens zur Autorisierung von Teilschritten veranlaßt werden. Ulbrichts Behutsamkeit war noch größer, wenn er sich dazu entschloß, ohne vorheriges Moskauer Einverständnis zu handeln. In jedem Fall war er bestrebt, die ihm gesetzten Grenzen nicht - oder wenigstens nicht allzu sehr - zu überschreiten. Wenn er den Kreml mit nicht vorbesprochenen Maßnahmen konfrontierte, ging er bis an die äußerste Grenze des vertretbar Erscheinenden. Er suchte nach außen hin die Solidarität mit dem Verbündeten selbst dann noch zu wahren, wenn er intern an seinem Verhalten Kritik übte. Es durften freilich nie Zweifel daran entstehen, daß die Entscheidungen über das Vorgehen gegenüber dem Westen in Moskau getroffen wurden. Die sowjetische Konfliktkontrolle war unbedingt aufrechtzuerhalten.

\section{Die Frage des politischen Einflusses der DDR}

$\mathrm{Zu}$ den Fragen, die Chruschtschow zu seiner Sache machte, gehörte die offene Westgrenze der DDR. Die Tatsache, daß er sich zweimal - 1958 und 1961 - nach vorherigem Widerstreben auf den Standpunkt stellte, den Ulbricht schon seit lan- 
gem eingenommen hatte, könnte die Annahme nahelegen, daß er dessen Druck nachgegeben habe. Die Quellen zeigen jedoch ein anderes Bild. Chruschtschow zeigte sich jeweils sehr lange von den Vorstellungen des SED-Chefs unbeeindruckt. Bis weit in das Jahr 1958 hinein war keine Rede davon, daß die offene Grenze ein Problem sei, das gelöst werden müsse, obwohl Ulbricht schon 1952 gefordert hatte, daß die Sperrmaßnahmen gegenüber der Bundesrepublik auf West-Berlin ausgedehnt werden müßten. Erst als der Kremlchef von sich aus zu dem Schluß gekommen war, daß die wirtschaftlichen Schwierigkeiten der DDR auf die Abriegelungslücke zurückzuführen seien, wurde diese zum Thema von Beratungen. Mit den Forderungen vom November 1958 zog Chruschtschow die Konsequenz aus seiner neuen Sicht. Bis Ende Juli 1961 gab er Ulbrichts zunehmendem Drängen kein Gehör, den Flüchtlingsstrom durch Schließung der Sektorengrenze rasch zu stoppen. Erst als er selbst zu der Auffassung kam, daß das Problem einer baldigen Lösung bedürfe und daß es keine Alternative zu Sperrmaßnahmen in Berlin gebe, wurden Vorbereitungen dazu eingeleitet. Wie Chruschtschow mit Ulbricht umsprang. wenn es ihm notwendig schien, zeigte sich in der Schlußphase der Berlin-Krise: Durch die erneute Anerkennung der Vier-Mächte-Rechte nahm er das Versprechen von 1958 zurück, den Anspruch der DDR auf unbeschränkte Souveränität gegen die Westmächte durchzusetzen. Ulbricht mußte sich fügen, fand sich aber nicht damit ab. ${ }^{1}$

Insgesamt konnte Ulbricht zwar versuchen, den Schwanz [die DDR] „kühn“ mit dem Hund [der UdSSR] wedeln zu lassen, ${ }^{2}$ aber der von Hope Harrison behauptete durchschlagende Erfolg 3 blieb ihm dabei versagt. Chruschtschow ließ sich nie zu einem Vorgehen drängen, das er nicht selbst für richtig oder wenigstens vertretbar hielt. Die Tatsache, daß die DDR 1961 vom Zusammenbruch bedroht war, veranlaßte ihn zwar dazu, die Hilferufe des SED-Chefs ernstzunehmen. Er war aber deswegen nicht bereit, dessen Vorstellungen zu folgen, wenn er anderes für besser hielt. Die Schließung der Grenze in Berlin, zu der er sich nach langem Zögern aufgrund eigenen Urteils entschloß, stand unter dem Vorbehalt, daß sie nur so lange aufrechterhalten werden sollte, wie die Massenflucht aus der DDR nicht durch Kontrolle über die West-Berliner Zugangswege unterbunden wurde. Anders als Ulbricht, der seinen Staat unbedingt vor systemfremden Einflüssen schützen wollte und ihn daher auch dann gegen West-Berlin abzuschirmen suchte, wenn dieses keine unkontrollierte Verbindung zum Westen mehr besaß, betrachtete er die Abriegelungsmaßnahme als ein bedauerliches Provisorium, das zu beenden war, sobald es eine andere Möglichkeit gab, die DDR-Bevölkerung im Lande zu halten. ${ }^{4}$ Daß die Sperranlagen bestehen blieben und zu einer festen Mauer wurden, lag nur daran, daß sein Bemühen um Kontrolle der Zugangswege

1 Vgl. die Äußerungen Ulbrichts auf seiner Internationalen Pressekonferenz, 19. 1. 1970, nach dem gesprochenen Wort wiedergegeben in: DDR-Spiegel (hrsg. vom Presse- und Informationsamt der Bundesregierung), 20.1.1970 (Sonderdienst), S. 26-28.

2 Hope M. Harrison, Driving the Soviets up the Wall. Soviet-East German Relations 1953-1961, Princeton/NJ-Oxford 2003, S. 139. Zur Sicht des Bemühens Ulbrichts um die Kontrolle der Sektorengrenze im Herbst 1960 siehe ebd., S. 144-148.

3 Vgl. ebd., S. 139-205, 218-221.

4 Aufzeichnung Chruščëvs, 11. 12. 1961, in: Istočnik, 6/2003, S. 125 f. 
aufgrund des Widerstands der Westmächte, vor allem der USA, nicht zum Erfolg führte.

Auch wenn sich Chruschtschow nicht von Ulbricht drängen ließ, hatte dieser wesentlichen Einfluß auf seine Politik. Nach Stalins Tod war er zum anerkannten Juniorpartner der Führungsmacht herangewachsen, der sich erhebliches Selbstbewußtsein leisten und seinen Standpunkt auch gegenüber dem Kreml vertreten konnte. Vor allem aber war er näher an den Problemen dran, um die es ging, so daß seinem Urteil in Moskau großes Gewicht zukam. Als Chruschtschow vor dem für ihn rätselhaften Phänomen stand, daß die DDR wirtschaftlich weit weniger florierte als die Bundesrepublik, obwohl sie doch das vermeintlich überlegene sozialistische System hatte, konnte ihm Ulbricht den Gedanken nahebringen, daß die offene Westgrenze zu wirtschaftlichen Störungen führe und dadurch die DDR in eine nachteilige Position versetze. Die Ansicht, daß Schließung der Grenze den innerdeutschen Systemwettbewerb von Beeinträchtigungen befreie, mag zwar dem Leser nicht einleuchten, doch kam für Chruschtschow kaum eine andere Erklärung in Betracht. Das Axiom, daß der Sozialismus weit höhere ökonomische Effizienz gewährleiste als der Kapitalismus, stand für ihn unumstößlich fest. Daher vermochte der sowjetische Führer nicht zu erkennen, daß die Misere der DDR hausgemacht war, die den Flüchtlingsstrom verursachte. Weder der restriktive Charakter des SED-Regimes noch Einzelmaßnahmen wie insbesondere die hastig durchgezogene Kollektivierung der Landwirtschaft kamen als Gründe für Not und Unzufriedenheit in Betracht, sondern allein die massiven Subversions- und Sabotageakte, die von West-Berlin aus ins Werk gesetzt würden. Wann immer Ulbricht seine Vorstellungen mit ideologischen Überzeugungen des Kremlchefs begründen konnte, befand er sich in einer starken Position.

\section{Verhältnis zur Bundesrepublik}

Die Bonner Entscheidung vom Frühjahr 1958, die Bundeswehr mit Kernwaffenträgern auszurüsten, um im Kriegsfalle nicht wehrlos dazustehen, signalisierte der sowjetischen Führung, daß sich ihre Hoffnung auf fortschreitende Distanzierung der Westdeutschen von der Politik der USA und der NATO nicht erfüllte. Von da an war die Bundesrepublik der Feind, den man durch heftige Polemik in den Augen der Welt diskreditieren mußte. Dem lag die Einschätzung zugrunde, daß sie für das westliche Bündnis unentbehrlich war: Von ihr hingen die amerikanische Europa-Präsenz, eine ausreichende territoriale Basis und die Verfügbarkeit wichtiger militärischer Ressourcen ab. Wenn es durch Ausübung von Druck doch noch gelang, diesen Beitrag zur atlantischen Allianz zu schmälern oder gar den Austritt aus der NATO zu erreichen, war ein Hauptziel erreicht. Die Erwartung, man könne zumindest Teilerfolge erzielen, wurde dadurch genährt, daß die Bundesrepublik aufgrund der deutschen Vergangenheit pschologisch-politisch besonders verletzlich war. Daher nahm der Kreml den propagandistischen Kampf gegen den „westdeutschen Revanchismus“ und „Militarismus“ auf. Die Feindseligkeit galt vor allem Adenauer und seiner Regierung, die den Kurs des Landes bestimmten. 
Als härtester Opponent der sowjetischen Berlin-Politik sah sich der Bundeskanzler sowjetintern dem Vorwurf ausgesetzt, daß er mit den westlichen Besatzungsrechten einen Anachronismus verteidige, der sich gegen die wachsende Macht des sozialistischen Lagers nicht länger aufrechterhalten lasse. Während in den westlichen Ländern die Einsicht wachse, daß man dem siegreichen Vordringen des Sozialismus im eigenen Interesse Rechnung tragen müsse, widersetze sich Adenauer mehr als alle anderen. Für Chruschtschow war der Bundeskanzler daher ein starrsinniger „alter Knacker“ (staryj chren), der hinter der Zeit zurückgeblieben war. Ihm müsse man daher beibringen, was die Stunde geschlagen habe. ${ }^{5}$

Nach sowjetischer Vorstellung hätte es der westdeutschen Staatsraison entsprochen, wenn man - vor allem aus ökonomischen Gründen - von den USA abgerückt wäre und sich der UdSSR angenähert hätte. Das hätte einen überaus attraktiven Absatzmarkt gewährleistet und die wirtschaftlichen Belastungen vermieden, die sich aus dem Wettrüsten der NATO ergäben. Während sich die Bundesrepublik im Westen einem scharfen Konkurrenzkampf gegenübersehe, werde ihr Verhältnis zur UdSSR von komplementären materiellen Bedürfnissen bestimmt. Damit bestünden die Voraussetzungen für einen beiderseits vorteilhaften Handel, der den Westdeutschen die Chance biete, ihre Verstrickungen im Westen zu lösen. Das gelte zwar nur auf staatlicher Ebene und lasse den Widerstreit der Systeme außer Betracht (den Chruschtschow nachdrücklich betonte), doch sei dies eine Streitfrage, die von der Geschichte entschieden werde und die daher für die aktuelle Politik keine Bedeutung habe. ${ }^{6}$ Die Bundesrepublik müsse daher „mit der aggressiven Politik der Revanchisten militaristischen Charakters im Verhältnis zum Osten brechen". Sie solle dabei weder eine neutrale Position beziehen noch die NATO verlassen und in den Warschauer Pakt eintreten, um bei anderen Führern in Westeuropa keine Ängste entstehen zu lassen, die deren Bindung an die USA zu festigen geeignet seien. Statt dessen hoffte Chruschtschow, die NATO durch Schaffung eines europäischen Sicherheitssystems zu eliminieren.7 Nach Ablösung Adenauers im Herbst 1963 glaubte Chruschtschow, die neue Regierung unter dem Wirtschaftsfachmann Erhard werde sich in diesem Sinne umorientieren. Der Besuch seines Schwiegersohnes Adshubej in Bonn Mitte 1964 sollte diese Entwicklung fördern.

\section{Die deutsche Frage}

Bei Chruschtschows Entschluß zur politischen Offensive gegen West-Berlin spielte die deutsche Frage eine wichtige Rolle. Das sehr populäre westliche Verlangen nach „Wiedervereinigung in Freiheit“ sollte durch ein attraktives Gegenprogramm gekontert werden. Deshalb wurde der alte, seit 1948 immer wieder in verschiedenen Formulierungen vertretene Standpunkt erneuert, die „Deutschen

5 Oleg Grinevskij, Tysjača odin den' Nikity Sergeeviča, Moskau 1998, S. 23-29.

6 Ebd., S. 122-128; Protokoll des Gesprächs Chruščëv - U Thant, 28. 8. 1962, in: Istočnik, 6/2003, S. $151 \mathrm{f}$.

7 Aufzeichnung Chruščëvs, 11. 12. 1961, in: Istočnik, 6/2003, S. 124 f. 
selbst“ müßten ohne „fremde Einmischung“ über ihre staatliche Einheit entscheiden. Die Forderungen nach „Überwindung der Überreste des Zweiten Weltkriegs“ und „Liquidierung des Besatzungsregimes“ sollten den Eindruck verstärken, die UdSSR setze sich für die Deutschen ein. Faktisch ging es jedoch darum, die Teilung zu vollenden. Wenn das Problem der nationalen Einheit der Entscheidung beider Staaten überantwortet wurde, war eine Einigung wegen der völlig gegensätzlichen Positionen von vornherein ausgeschlossen. Die geforderte Umwandlung West-Berlins in eine „Freie Stadt“ von östlichen Gnaden zielte darauf $\mathrm{ab}$, den letzten Restbestand der früheren deutschen Einheit zu beseitigen, der nicht zur Logik der staatlichen Trennung paßte.

Der Kreml betonte zu recht, die Lage in der geteilten Stadt Berlin sei „anomal“ und erfordere eine „Normalisierung“. Zwar war nach dem Bau der Mauer WestBerlin nicht mehr der frühere „Pfahl im Fleische“ der DDR und des sowjetischen Imperiums, blieb aber der „Pfahl im Fleische“ der deutschen Teilung. Das Scheitern des Versuchs, diesen zu beseitigen, bestimmte auf entscheidende Weise den weiteren Verlauf des Ost-West-Konflikts und die weitere Entwicklung der deutschen Frage. Der westliche Schutz für West-Berlin blieb erhalten. Die Anomalie seiner Existenz inmitten einer feindlichen DDR bestand fort. Sie wurde nicht gemäß den Wünschen Chruschtschows und Ulbrichts durch Schaffung eines festen politischen und rechtlichen Fundaments für die Zweistaatlichkeit beseitigt. Statt dessen blieb die Option offen, sie durch Wiederherstellung der deutschen Einheit zu überwinden. Die Tatsache, daß die Teilung nicht als durchgängige Normalität durchgesetzt, sondern durch eine weiterbestehende Anomalie gestört wurde, machte es möglich, daß es 1989/90 zur Wiedervereinigung kam, die nicht zufällig ihren Ausgang von Berlin aus nahm. Eingeleitet wurde sie durch den Fall der Mauer, die Chruschtschow wegen der nicht durchgesetzen Kontrolle über WestBerlin autorisiert hatte und die seitdem die Anomalie der staatlichen Trennung mehr als alles andere augenfällig gemacht hatte.

\section{Die Bedeutung des militärischen Kräfteverhältnisses}

Chruschtschow verband seine Vorstellung eines freien Wettbewerbs zwischen den Systemen in den ausgehenden fünfziger Jahren mit der Idee eines weithin entmilitarisierten Ost-West-Verhältnisses. Zwar war er sich grundsätzlich der Bedeutung bewußt, die den Streitkräften und Rüstungen in den internationalen Beziehungen zukam, doch glaubte er, auf die Dislozierung sowjetischer Soldaten im Vorfeld der UdSSR verzichten zu können. Anders als Stalin, der eine langdauernde Besetzung Deutschlands für notwendig erachtet und nur aus Propagandagründen das Gegenteil erklärt hatte, wollte er tatsächlich seine Streitkräfte vom Territorium anderer Staaten abziehen, wenn sich die Westmächte ebenfalls dazu bewegen ließen. Damit, so hoffte er, werde der Weg frei zur Entfernung der USA aus Europa und zum Zerfall der NATO. Die Sicherheit auf dem Kontinent sollte nicht mehr durch gegeneinander gerichtete Bündnisse, sondern durch ein kollektives Sicherheitssystem gewährleistet werden, an dem sich die europäischen Staaten von Ost und West beteiligten. Der UdSSR wäre notwendig die Rolle der Führungsmacht zu- 
gefallen; das Kräfteverhältnis hätte sich entscheidend zu ihren Gunsten verändert.

Die Bereitschaft zum Verzicht auf die militärische Präsenz in Ostmittel- und Südosteuropa war zum einen dadurch motiviert, daß die UdSSR durch ihre weitreichenden Kernwaffen vor feindlichen Angriffen geschützt war und daher zu ihrer Verteidigung keine Truppen außerhalb ihrer Grenzen benötigte. Zum anderen war Chruschtschow davon überzeugt, daß die kommunistischen Parteien in den ostmittel- und südosteuropäischen Nachbarstaaten den Bestand und den Zusammenhalt des sozialistischen Lagers ausreichend gewährleisteten. Die Krise der DDR von 1953 schien durch Vorkehrungen zur Bekämpfung der „Konterrevolution" überwunden, und auch die Lage in Polen und Ungarn hielt er nach den Eruptionen des Jahres 1956 für stabilisiert. Im übrigen führte er die Unzufriedenheit der „Bruderländer“ auf den offensichtlichen Mangel an Gleichberechtigung zurück, der in der Stalin-Zeit und in den folgenden Jahren das Verhältnis der UdSSR zu ihren Verbündeten belastet hatte. Er suchte dem Übel durch weitreichende Zugeständnisse an den Souveränitätsbedarf der Partner abzuhelfen. Seine Ankündigung vom November 1958, die DDR in ihre vollen Rechte einzusetzen, ist auch in diesem Kontext zu sehen. Wenn die UdSSR die Selbständigkeit der anderen Staaten respektiere, werde sich der sozialistische „Bruderbund“ auf der Grundlage der Freiwilligkeit festigen und daher keine militärische Klammer benötigen.

Nicht Zweifel an der politischen Festigkeit der kommunistischen Regimes in Ostmittel- und Südosteuropa, sondern das westliche Verhalten bewog Chruschtschow Anfang der sechziger Jahre zur Kursänderung. Die Westmächte waren keinesfalls zum Verzicht auf die Stationierung ihrer Truppen in der Bundesrepublik bereit und ließen sich von den Kernwaffen der UdSSR weniger beeindrucken als erhofft. Chruschtschow stellte zwar den Regierungschefs in Westeuropa, etwa Macmillan oder Adenauer, immer wieder vor Augen, er könne ihre Länder mit einem einzigen Nuklearschlag vernichten, aber die entscheidende Macht im Westen, die USA, war durch solche Drohungen nicht zu beeindrucken. Das sowjetische Arsenal bestand fast ausschließlich aus Mittelstreckenraketen, die sich nicht gegen Nordamerika einsetzen ließen. Umgekehrt jedoch befand sich die Sowjetunion in der Reichweite vieler amerikanischer Interkontinentalsysteme. $8 \mathrm{Ab}$ 1960/61 kam Chruschtschow zu der Ansicht, die Ablehnung seiner Berlin-Forderungen sei darauf zurückzuführen, daß man in Washington die militärischen Fähigkeiten seines Landes, nicht zuletzt auch im konventionellen Bereich (um dessen Reduzierung er bis dahin bemüht gewesen war), für schwach hielt. Er suchte daraufhin diesen Mangel durch Verstärkung vor allem der Truppen auf dem potentiellen Gefechtsfeld und durch Aktivierung des bis dahin vernachlässigten Warschauer Pakts zu beheben. Fortan galt, daß das militärische Potential gegenüber dem Westen nicht nur auf nuklearstrategischer Ebene, sondern auf allen Ebenen der Vergrößerung bedürfe.

8 Vgl. Andreas Wenger, Living with Peril. Eisenhower, Kennedy, and Nuclear Weapons, LanhamBoulder-New York-Oxford 1997, S. 145-237. 
Der Aufbau einer mächtigen Drohkapazität schien insbesondere im Blick auf die Konfrontation wichtig, die beim vorgesehenen Abschluß des separaten Friedensvertrags zu erwarten war. Weder die einsetzenden Bemühungen um Truppenverstärkung noch die Explosion der Superbombe im Herbst 1961 veranlaßten jedoch die USA zum erhofften Nachgeben. Im Oktober 1962 scheiterte der Versuch Chruschtschows, durch die Aufstellung von Raketen auf Kuba das strategische Kräfteverhältnis zu ändern. Der Kremlchef mußte erkennen, daß die Politik der Androhungen, die er nicht wahrmachen wollte und konnte, ein höchst gefährliches Spiel mit dem Feuer war. Zudem stand sie in Widerspruch zu wichtigen politischen Zielen. Selbst in Zeiten akutester Krise war er stets an guten Beziehungen zu den USA interessiert. Er wollte keine Konfrontation mit der anderen Supermacht, hoffte auf Vorteile einer wirtschaftlich-technischen Kooperation und bemühte sich um Abrüstungsvereinbarungen, um im Interesse der Wohlfahrt seines Landes die Militärausgaben zu beschränken, ohne Nachteile im Kräfteverhältnis zu den Amerikanern zu haben. Erst nachdem die sowjetische Drohposition in der Kuba-Krise zusammengebrochen war, entschloß er sich, rückte er dieses Ziel, das er bis dahin in den Beziehungen zu den Vereinigten Staaten mehr theoretisch als praktisch im Auge gehabt hatte, in den Vordergrund seiner Westpolitik. Im Blick auf Berlin hieß dies, daß die offensiven Ziele bis auf weiteres in den Hintergrund zu treten hatten, weil das auf anderen Feldern angestrebte Zusammenwirken mit den USA nicht gestört werden durfte. 